\title{
Florid Cemento-Osseous Dysplasia
}

\author{
Usha Asnani $^{1}$, Jyotsna S. Galinde ${ }^{2}$, Srivalli Natrajan ${ }^{3}$, Mahesh Sonar ${ }^{4}$
}

\begin{abstract}
Florid cemento-osseous dysplasia (FCOD) is an uncommon benign multifocal dysplastic lesion affecting the jaws that consists of cellular fibrous connective tissue with bone and cementum like tissue. It is most commonly seen in middle aged black woman although it can occur in Caucasians and Asians. A case of FCOD in a 63 year old Indian woman with unique radiographic and clinical presentation is reported.
\end{abstract}

Key words: FCOD, Fibroosseous dysplasia, cemento-osseous dysplasia.

\section{Introduction:}

Florid cemento-osseous dysplasia, periapical cementoosseous dysplasia, and focal cemento-osseous dysplasia have been designated by the World Health Organization as cemento-osseous dysplasias $(1,2)$. The cause of these lesions is unknown ${ }^{3}$. Classification of cemento-osseous lesions of the jaws has long been a complex and controversial dilemma for pathologists ${ }^{4}$. Currently, no universally accepted classification exists. A large group of these lesions exhibits sufficiently distinctive clinico-radiographic and histologic features to be separated into a specific category labelled as florid cemento-osseous dysplasia (FCOD). These cases have been reported under a variety of diagnoses including multiple cemento-ossifying fibromas, sclerosing osteitis, multiple enostosis, multiple osteomas, periapical cementoblastoma, Paget's disease of the mandible, gigantiform cementom $\mathrm{a}^{5}$, chronic sclerosing osteomyelitis ${ }^{4}$, sclerotic cemental masses of the jaws, and multiple periapical osteofibromatosis. Non-epithelium lined cyst formation associated with FCOD was first reported by Melrose et $\mathrm{al}^{6}$ in 1976 and followed by several reports.

The current classification of cementooseous lesions, formulated in 1992 by the World Health Organization $(\mathrm{WHO})^{7}$, is based on age, sex, and histopathologic, radiographic, and clinical characteristics, as well as location of the lesion. This classification includes cemento- ossifying fibroma, benign cementoblastoma, and the group of the cemento-osseous dysplasias.

1 Professor

2 Professor \& HOD

3 Professor

4 Reader

Address for Correspondence :

Dr. Usha Asnani

Dept of Oral \& Maxillofacial Surgery

MGM Dental College \& Hospital, Kamothe, Navi Mumbai.

Mob.: 9820897181

E-mail: drushaasnani@gmail.com
Periapical cemental dysplasia and florid cementoosseous dysplasia, which have also been reported under the term "gigantiform cementoma ${ }^{8}$,"are included in the latter group. This disorder appears to be limited to the jaws and is often asymptomatic. The gigantiform cementoma or florid cementoosseous dysplasia, usually affects middle aged black women. It is a benign fibro-osseous lesion, typically involving multiple sites and frequently symmetric in distribution. It is not unusual to find extensive lesions in all 4 posterior segments of the jaws ${ }^{4}$ and is a more extensive manifestation of the same lesion present in periapical and focal cemento osseous dysplasia ${ }^{4}$. When the lesions are large, jaw expansion and facial deformity may be apparent. The lesions have an avascular nature.

Radiographically, a mixed radiolucent and radiopaque appearance is the common finding. The lesions appear lobular and irregularly shaped and progress from radiolucent to radiopaque lesions over time. It is common to note these lesions in all four quadrants of the jaws ${ }^{(2,9,10)}$. Involved areas of the jaw may undergo expansion.

Histologically, the radiopaque lesions that are present appear to consist of an osteoid and cementum-like material, with fibroblasts being associated with the trabeculae of the calcified material ${ }^{2}$. Simple bone cysts and inflammatory cells may also be associated with the lesions. Unless symptoms are noted, no treatment is normally rendered. Once infected the patient become symptomatic, and treatment of the secondary infection is very difficult, and antibiotics are often not effective ${ }^{(9,10)}$.

\section{Case Report:}

A 63 years old female reported to the department of oral and maxillofacial surgery with swelling in the upper jaw since 4 to 5 years[fig 1 \&2]. History revealed that the swelling had started in left upper posterior 


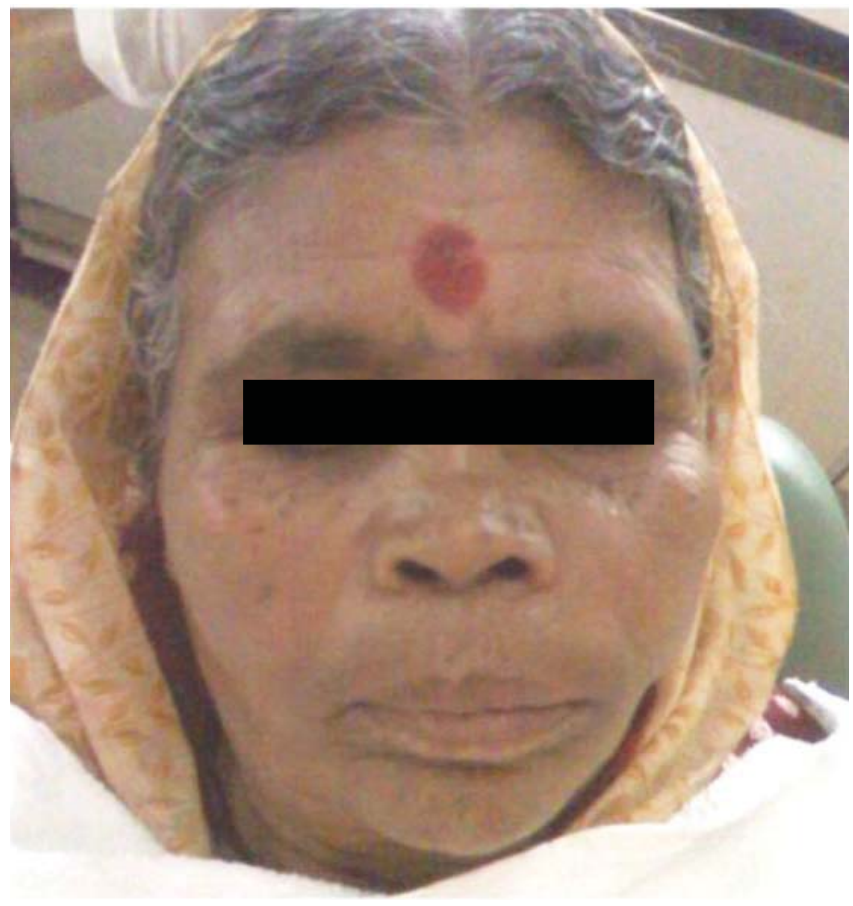

f Fig 1: Clinical photograph of the patient showing swelling on the left side of the cheek.

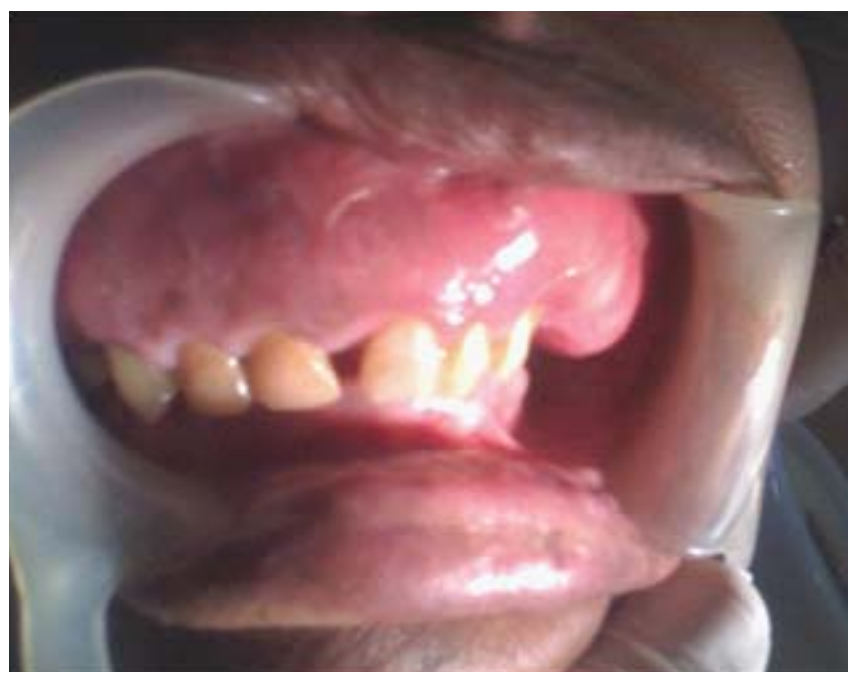

Fig 2: Intraoral photograph of the patient showing lobulated mass and buccal expansion in the maxilla.

region which progressively increased to involve the anterior region and extended upto the tuberosity on right side. History of spontaneous exfoliation of few teeth in the involved area and intermittent episodes of sinuses with pus discharge was present.

Extraoral examination revealed that the patient had prominent malar bones and frontal bossing. Swelling was present in the maxillary alveolar region extending from left tuberosity to right buttress region. Buccal and palatal expansion was present. Surface appeared lobulated with overlying mucosa of normal color and texture. Premolars on left side showed displacement. Molar teeth on left side were missing. On palpation the swelling was hard and nontender. Involved teeth were found vital.

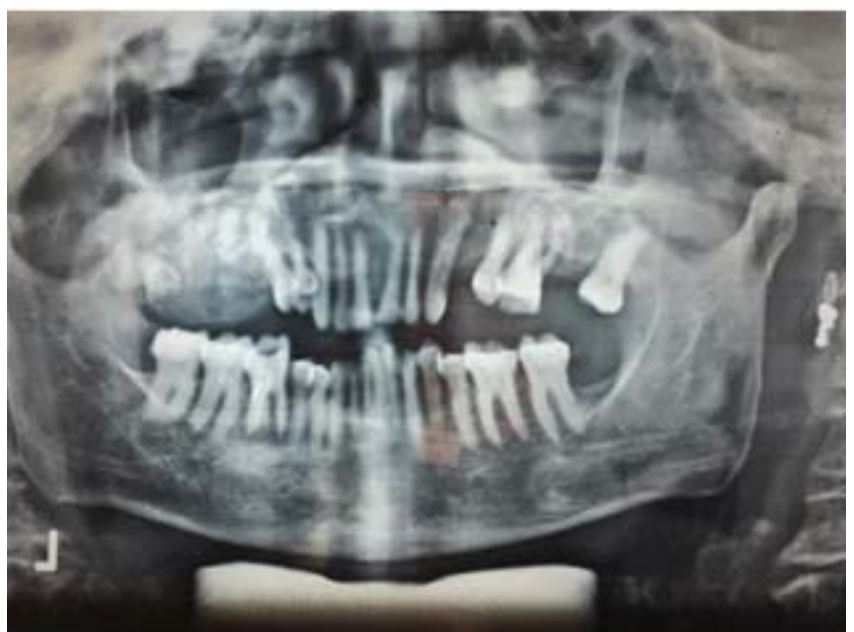

Fig.3: OPG shows irregular radiopacities mixed with poorly defined radiolucency from upper left third molar to right tuberosity

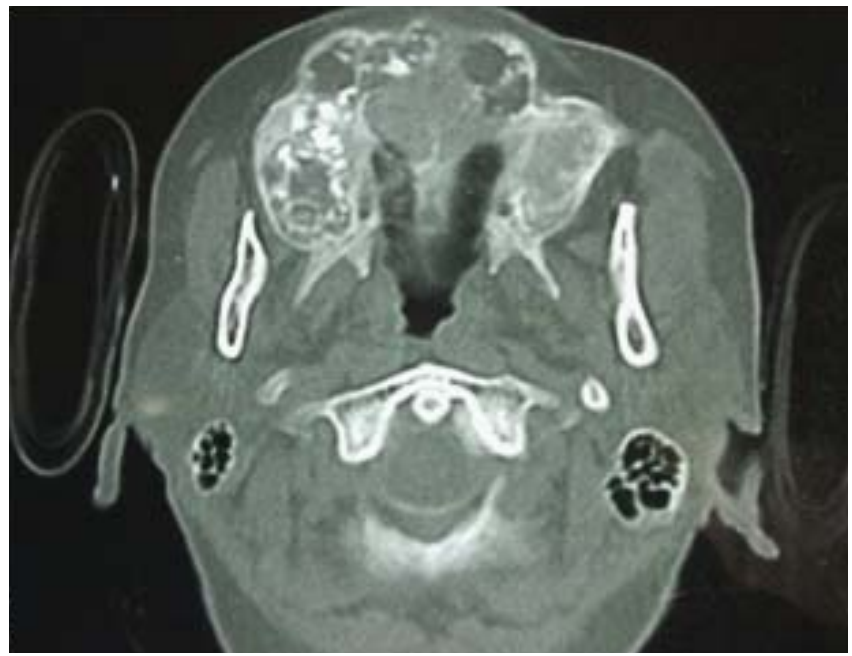

Fig 4: Axial CT showing mixed hypo \& hyperdense masses extending from right tuberosity to left tuberosity with Buccal \& Palatal expansion

Orthopantamogram [fig 3] revealed irregular radiopacities in tooth bearing alveolar areas mixed with the poorly defined radiolucency from left third molar to right first molar. Most of involved teeth showed hypercementosis. Masses appeared to be separated from root apices. The maxillary sinus and mandible did not show any involvement.

Computed tomography (CT) axial and coronal sections revealed poorly defined mixed hypo and hyperdense masses, with cloud-like appearance involving maxilla extending from right to left tuberosity[fig 4\&5]. There was buccal and palatal expansion and the lesion showed lobulated surface. Coronal sections revealed that the maxillary sinuses were normal with minimal fluid levels. These sections also showed the classic ground glass appearance of the malar bones, bones of the cranial vault including the parietal and temporal bones. However, the patient had no history of headache or signs of cranial nerve involvement. The blood chemistry showed that serum alkaline 


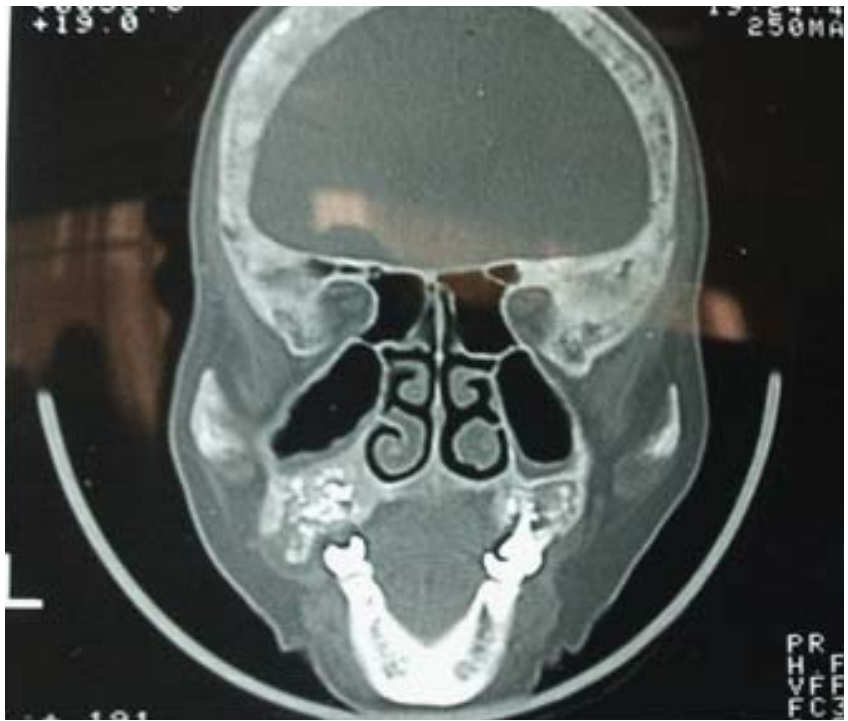

Fig.5: Coronal CT showing ground glass appearance with thickening of bones of cranial vault, malar bones \& mixed radiolucency \& radiopacity in the buttress region

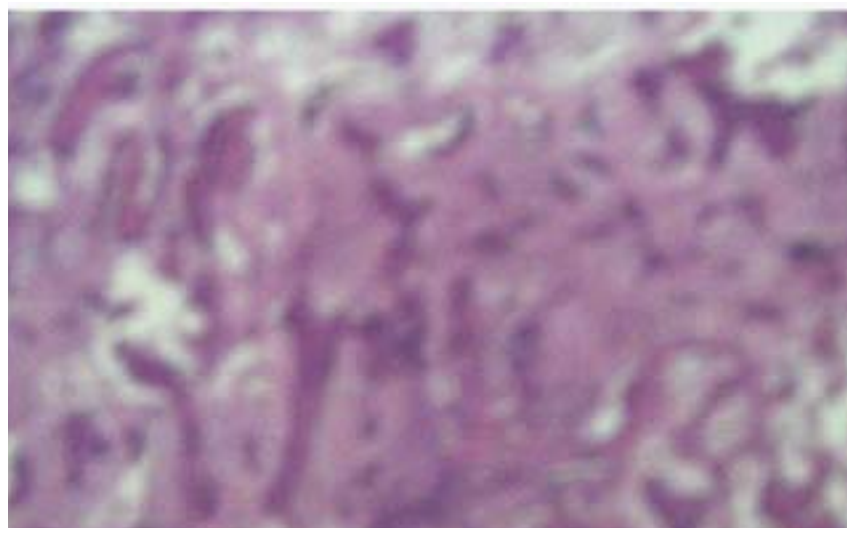

Fig.6: Cementum like substance with relatively acellular structure are scattered among areas of fibrous stroma.

phosphatase was raised [1469U/L]. Incisional biopsy was done. Histopathologically the tissue showed mixture of woven bony trabeculae and cementum like calcifications with areas of loose fibrocollagenous stroma. Osteoblastic rimming and reversal lines were seen in bony trabeculae. The stroma between the islands of cementum like substance was poorly vascularized [fig 6]. The microscopic findings were compatible with the diagnosis of FCOD.

Though CT findings suggested the possibility of a craniofacial fibrous dysplasia occurring concomitantly with FCOD, considering the extensive nature of involvement of craniofacial fibrous dysplasia and the lack of the symptoms, it was decided to address only the FCOD component. The latter lesion showed recurrent episodes of infection and progressive expansion warranting surgical excision. Mucoperiosteal flap was raised and the lesion with involved teeth was excised form the left tuberosity to right first molar region. The bone was found to be soft, vascular and was interspersed with areas of calcification. As the lesion showed no well defined boundaries, care had to be taken during excision to ensure that the floor of the maxillary sinus was not breached. Postoperative healing was uneventful and histopathology report confirmed the diagnosis of FCOD. Patient after one year of follow up showed good healing and no evidence of recurrence.

\section{Discussion:}

Florid cemento-osseous dysplasia is a rare benign lesion arising from elements of the periodontal ligament 4 and is strictly localized to the tooth bearing areas. Many lesions have to be differentiated from FCOD, and dental imaging can be used to discriminate between FCOD and other lesions that may exhibit a similar sclerotic appearance on conventional radiographs. Odontogenic tumors, especially cementoossifying fibroma, generally exhibit more buccolingual expansion than does FCOD11. Dental imaging may be helpful in differentiating fibro-osseous lesions from odontoma, in which the CT number for enamel is higher than that for cementum. Cementoblastoma characteristically is fused to the tooth apices. In hypercementosis, the cemental substance lies in continuity with the dental root, whereas in florid cemento-osseous dysplasia or gigantiform cementoma, it is separated from the periodontal space. This latter feature is clearly evident in the radiograph of this patient. Differential diagnosis of FCOD must also include sclerosing osteomyelitis, which can be a complication of the disease ${ }^{12}$. However etiopathogenesis is not clear, Some authors attribute the pathogenesis of FCOD to the proliferation of the fibroblasts present in the periodontal ligament, while, few think that it may arise from the remnants of the cemetum left after the extraction of the teeth. Some contribute the etiologic factors to the slight trauma, such as deepbite and trauma from occlusion ${ }^{13}$. Waldron et al. ${ }^{14}$ have proposed that reactive or dysplastic changes in the periodontal ligament might be a cause for the disease. Radiographic appearance depends upon the stage of development and maturity of the lesion. At proliferative stage, lesion appears radiolucent and in the later stages it appears as dense radiopaque masses appearing at the apices of teeth or tooth bearing posterior region of mandible and maxilla. Histopathologically , FCOD is composed of a proliferating fibrous connective tissue stroma containing foci of cementum along with the presence of osteoid or bone. More advanced lesions show an increase in mineralization. In FCOD large sclerotic masses are formed that are hypocellular and extremely dense with small marrow spaces and few haversian systems.

In our case the diagnosis of FCOD was made clinco- 
radiologically and histopathologically. Possibly because of spontaneous exfoliation of teeth, sclerotic masses were exposed to the oral cavity resulting in recurrent infection and pus discharge. This case was unusual as there was presentation of concomitant Craniofacial fibrous dyslplasia and FCOD but as the former component was asymptomatic, patient was kept under observation for the same.

The management of FCOD is twofold. In asymptomatic patients it is probably wise to keep the patient under observation without surgical intervention 4 . Because the onset of symptoms is usually associated with exposure of the sclerotic masses to the oral cavity, biopsy or elective extraction of teeth in the involved area should be avoided. It would be impractical to completely resect the lesion because it usually occupies most of the mandible and maxilla. When surgical intervention is indicated, a remodelling resection is recommended for esthetic reasons. When the patient is symptomatic secondary to a tooth pain, the tooth may be managed endodontically by avoiding extractions. Cases with secondary predisposed factor of infection are difficult and complicated to manage.

\section{References:}

1) Kawai T, Hiranuma H, KishinoM, Jikko A, Sakuda M. Cemento-osseous dysplasia of the jaws in 54 Japanese patients: a radiographic study. Oral Surg Oral Med Oral Pathol Oral Radiol Endod. 1999; 87:107-14.

2) $\mathrm{Su} \mathrm{L}$, Weathers DR, Waldron CA. Distinguishing features of focal cemento-osseous dysplasias and cemento-ossifying fibromas, I: a pathologic spectrum of 316 cases. Oral Surg Oral Med Oral Pathol Oral Radiol Endod. 1997; 84:301-9.

3) Beylouni I, Farge P, Mazoyer JF. Florid cemento-osseous dysplasia: report of a case documented with computed tomography and 3D imaging. Oral Surg Oral Med Oral Pathol Oral Radiol Endod. 1998; 85:707-11.

4) Waldron CA. Fibro-osseous lesions of the jaws. J Oral Maxillofac Surg. 1993; 51:828.

5) Van Der Wall I, Van Der Kwast WAM. A case of gigantiform cementoma. Int J Oral Surg 1974; 3: 440-4.

6) Melrose RJ, Abrams AM, Mills BG: Florid osseous dysplasia: A clinical pathologic study of thirty-four cases. Oral Surg Oral Med Oral Pathol. 1976;41:62.

7) Alberto Toffanin, Roberto Benetti, Riccardo Manconi, Familial florid cemento-osseous dysplacia: a case report. J Oral Maxillofac Surg 2000; 58:1440-1446.

8) Waldron CA. Fibro-osseous lesions of the jaws. J Oral Surg. 1985; 43:249.

9) Neville BW, Damm DD, AllenCM, Bouquot JE. Oral and maxillofacial pathology. 1st ed. Pennsylvania: WB Saunders Co. 1995:464-8.

10) Bencharit S, Schardt-Sacco D, Zuniga JR, Minsley GE. Surgical and prosthodontic rehabilitation for a patient with aggressive florid cementoosseous dysplasia: a clinical report. J Prosthet Dent. 2003; 90:220-4.

11) DelBalso AM, Werning JT. The role of computed tomography in the evaluation of cemento-osseous lesions. Oral Surg Oral Med Oral Pathol. 1986; 62:354-7.

12) Shafer WG. Chronic sclerosing osteomyelitis. J Oral Snrg. 1957; 15:138-42.

13) Reichart PA, Philpsen HP, Odontogen Tumors and Allied Lesions. Unilessence Publ. London. 2004; 35:301-308.

14) Loh FC, Yeo JF. Florid osseous dysplasia in Orientals. Oral Surg Oral Med Oral Pathol. 1989 Dec; 68(6):748-53. 